\title{
Peste, uma zoonose esquecida
}

\section{Plague, a forgotten zoonosis}

\author{
Celso Tavares ${ }^{1,2}$ (D) , Nilma Cintra Leal ${ }^{3}$ (D) , Marise Sobreira ${ }^{3}$ (D) Alzira Maria Paiva Almeida $^{3}$ (C)
}

1. Câmara Técnica de Infectologia do Conselho Regional de Medicina de Alagoas (CRM-AL) e do Conselho Federal do Medicina (CFM). 2. Assessor da Secretaria de Saúde de Alagoas, Maceió, AL, Brasil. 3. Serviço de Referência Nacional de Peste do Instituto Aggeu Magalhães (IAM), FIOCRUZ, Recife, PE, Brasil.

\section{Resumo}

Objetivo: caracterizar aspectos epidemiológicos e clínicos fundamentais, discutir a metodologia do diagnóstico e tecer recomendações sobre as condutas perante a suspeição de casos de peste. Métodos: revisão bibliográfica e levantamento das internações e mortes por peste registradas no Sistema de Informações Hospitalares do Sistema Único de Saúde. Resultados e conclusões: a existência de diagnósticos equivocados de uma doença potencialmente fatal, além dos registros hipotéticos de internações e mortes, constitui um desafio a ser superado, pois espelha uma situação inaceitável, em que um possível e insólito diagnóstico não é investigado e acumula-se nos sistemas de informação.

Palavras-chave: Peste. Yersinia pestis. Focos naturais. Epidemiologia. Diagnóstico.

\begin{abstract}
Objective: to characterize ground epidemiological and clinical aspects of, discuss the methodology of diagnosis and draw recommendations about the management of suspect cases of plague. Methods: literature review and data collection of hospitalizations and deaths due to plague recorded in the Hospital Information System of the Unified Health System. Results and conclusions: the existence of mistaken diagnoses of a potentially fatal disease, as well as hypothetical records of hospitalization and deaths, is a challenge to be overcome, because it reflects an unacceptable panorama in which a possible and unusual diagnosis is not investigated and accumulates in the information systems.
\end{abstract}

Keywords: Plague. Yersinia pestis. Natural foci. Epidemiology. Diagnosis.

\section{INTRODUÇÃO}

A peste aflige a humanidade desde sempre, exercendo infinita influência no imaginário popular ${ }^{1}$. A zoonose, primariamente de roedores e de transmissão vetorial, é endêmica em certos países na África, Ásia, antiga União Soviética e nas Américas, onde persistem numerosos focos naturais ${ }^{2}$. No Brasil, as áreas focais situam-se nas Serras da Ibiapaba e do Baturité (Ceará), Chapada do Araripe (Ceará, Pernambuco e Piauí), Chapada da Borborema (Alagoas, Paraíba, Pernambuco e Rio Grande do Norte), Serra de Triunfo (Paraíba e Pernambuco), Planalto Oriental, Chapada Diamantina e seu piemonte (Bahia), Vales do Rio Doce e do Jequitinhonha (Minas Gerais) e Serra dos Órgãos (Rio de Janeiro) ${ }^{3}$. Esses focos são remanescentes da introdução da infecção pelo porto de Santos, SP durante a última pandemia em 1899 (figura 1). Até a década de 1980, ocorriam surtos a cada 5 - 10 anos, que eram entremeados por períodos variáveis de quiescência ${ }^{4}$; porém, a maioria das áreas focais não apresenta notificações há mais de 50 anos, apesar da detecção da circulação da Yersinia pestis, o seu agente etiológico, em animais sentinela ${ }^{5,6}$. Desde 2005, casos suspeitos isolados são notificados, mas todos eles têm sido, sistematicamente, descartados laboratorialmente ${ }^{5,6}$.

A peste, à luz dos conhecimentos atuais, não poderá ser erradicada dos seus focos naturais ${ }^{7}$, ainda conserva seu caráter atemorizante, e qualquer suspeita de um novo caso pode desencadear pânico, mesmo entre os profissionais de saúde. A seu famigerado estigma, acrescente-se que a Y. pestis está categorizada na classe 3 de risco biológico e no grupo A dos agentes de bioterrorismo ${ }^{1,8}$.

Assim, a divulgação pelos meios de comunicação de um caso suspeito de peste no município de São Gonçalo, RJ, em janeiro de 2019, gerou momentâneo interesse na imprensa, com a divulgação de um "release" por todas as mídias.

A ocorrência desse putativo caso de peste e a existência de 74 registros espúrios de mortes e de 998 de internação no Sistema de Informação Hospitalar do Sistema Único de Saúde (SIH/SUS), no período de 2007 a 2019, levaram à realização desta revisão bibliográfica, objetivando caracterizar aspectos epidemiológicos da zoonose, discutir a metodologia do diagnóstico e tecer recomendações sobre as condutas perante a suspeição de casos.

A peste, atualmente, é uma "zoonose esquecida" e, portanto, o nível de conhecimento da maioria dos profissionais de saúde é

Correspondência: Alzira Maria Paiva Almeida. Campus da UFPE - Av. Prof. Moraes Rego, s/n - Cidade Universitária, Recife - PE, 50670-420. E-mail: aalmeida@cpqam.fiocruz.br

Conflito de interesse: Não há conflito de interesse por parte de qualquer um dos autores.

Recebido: 16 Maio 2019; Revisado: 25 Out 2019; Aceito: 6 Dez 2019 
insuficiente perante a ocorrência de casos humanos isolados e, consequentemente, a prevenção e o controle de epidemias ${ }^{9,10}$. Esse desconhecimento, por sua vez, também possibilita que pacientes com outros agravos, inclusive de natureza não infecciosa, sejam considerados casos suspeitos, mesmo sem apresentarem quaisquer comemorativos epidemiológicos ou manifestações clínicas da infecção.

Figura 1. Principais áreas focais da peste no Brasil.

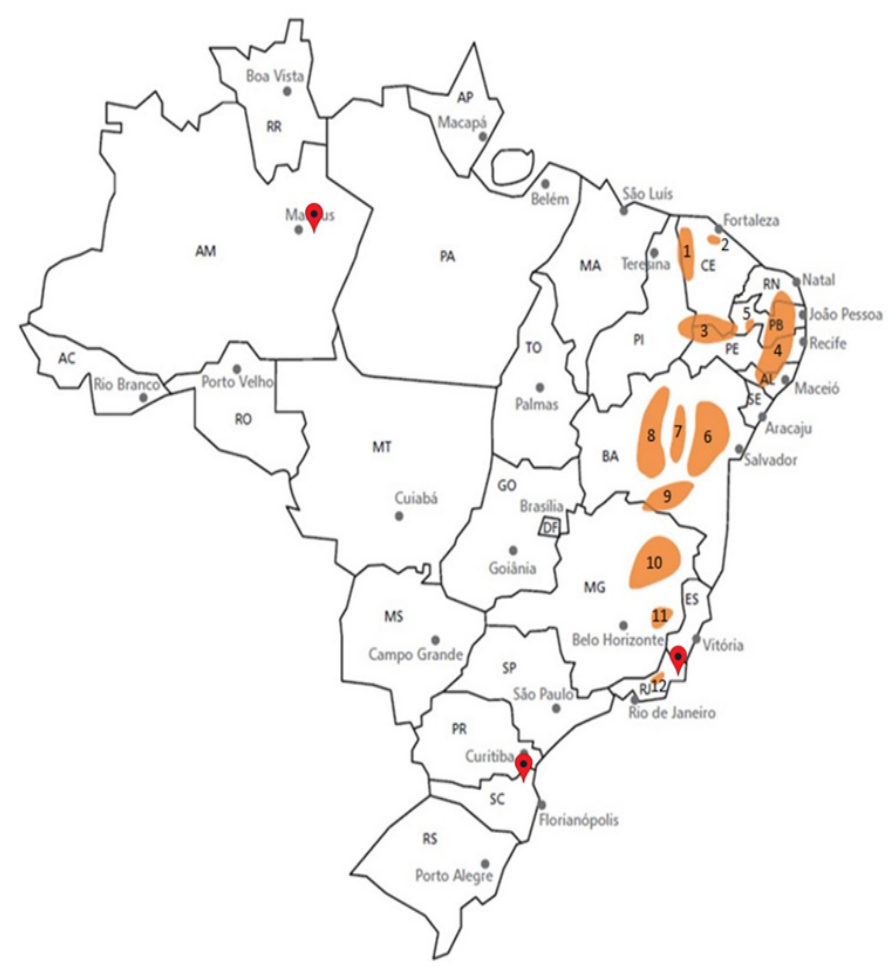

Legenda: 1: Serra da Ibiapaba, 2: Serra de Baturité, 3: Chapada do Araripe, 4: Chapada da Borborema, 5: Triunfo, 6: Planalto Oriental, 7: Piemonte da Diamantina, 8: Chapada Diamantina, 9: Planalto da Conquista, 10: Vale do Jequitinhonha, 11: Vale do Rio Doce, 12: Serra dos Órgãos, e a localização dos casos de falso diagnóstico em áreas indenes $\%$.

O diagnóstico de peste requer que os profissionais responsáveis pelas assistências secundária e terciária tenham pleno conhecimento de seus caracteres epidemiológicos, clínicos e terapêuticos, pois alguns casos evoluirão desfavoravelmente ou com apresentações clínicas que exigirão cuidados especiais ${ }^{11}$.

O espectro da infecção pestosa é abrangente, desde formas assintomáticas a quadros rapidamente letais. Clinicamente, dependendo do modo de transmissão e das relações patógeno $x$ hospedeiro, a infecção pode ser oligossintomática, manifestarse em suas apresentações mais prevalentes - a bubônica, a septicêmica e a pneumônica ou ainda em formas infrequentes, como a cutânea, a tonsilar, a ocular, a gastrointestinal e a meníngea ${ }^{11}$. O conhecimento dos aspectos epidemiológicos, clínicos e laboratoriais é essencial para o diagnóstico. Assim, comemorativos epidemiológicos primordiais na avaliação de quaisquer casos suspeitos devem ser enfatizados à anamnese - local de residência e trabalho, ocorrência de epizootias, viagens recentes, inclusive, a outros países, nos últimos 12 dias, contatos com pessoas e animais sadios ou doentes procedentes dos focos e da profissão, especialmente aquelas que lidam com animais, sobretudo, os de estimação $0^{1,11,12}$. O conhecimento que a peste é uma zoonose focal e de todas as implicações que essa condição acarreta certamente favorece o diagnóstico.

Na vigência de casos de pneumonia pestosa em área urbana, a hipótese de uma ação de bioterrorismo ${ }^{8}$, apesar de improvável no Brasil, deverá ser devidamente avaliada; porém, a hipótese mais provável será a de casos secundários decorrentes da falta de diagnóstico de uma doença febril aguda com grande comprometimento sistêmico ou da má assistência prestada a doentes com quaisquer formas da peste ${ }^{6,11}$.

As equipes dos laboratórios de análises clínicas também devem ser, sistematicamente, atualizadas, o que evitará equívocos diagnósticos. Um procedimento que pode induzir ao erro, desde que o protocolo vigente não seja observado, é a utilização de sistemas automatizados de identificação microbiana pelos laboratórios de análises clínicas ${ }^{13}$. Pode constituir um problema de consequências desastrosas para o paciente, a comunidade e o Estado se os resultados referentes à $Y$. pestis não forem devidamente validados, pois tendem a confundir outras espécies da família Enterobacteriaceae e, principalmente, os organismos não fermentadores com o bacilo pestoso ${ }^{1,13}$. Desse modo, é mandatório que todos os isolados identificados como $Y$. pestis em laboratórios de análises clínicas de todo o país sejam encaminhados aos laboratórios de referência, onde serão submetidos às provas que confirmarão ou descartarão o diagnóstico ${ }^{3,14}$.

O uso crescente desses sistemas no País elevou o número de casos diagnosticados como peste ( $Y$. pestis), sem que, até o momento, a hipótese se tenha confirmado. No caso de São Gonçalo, o diagnóstico não teve fundamentação epidemiológica e clínica e esteou-se, exclusivamente, na identificação da $Y$. pestis em cultura realizada no laboratório do hospital e que não foi confirmada pelas provas específicas de identificação $0^{3,14}$. Outros casos que não receberam tanta notoriedade foram registrados nas cidades de Manaus (AM) e Jaraguá do Sul (SC), áreas indenes (figura 1). A identificação da $Y$. pestis em amostra biológica de pacientes humanos ou de origem animal deverá ser confirmada por análises bacteriológicas que determinarão as características tintoriais, culturais, bioquímicas e antibióticoresistência e a caracterização molecular do isolado ${ }^{1,3,14}$.

O registro indevido de mortes e internações no SIS/SUS, por sua vez, necessita ser devidamente avaliado para que se conheçam as causas que justificaram a inclusão da CID A20 em Autorização de Internação Hospitalar (AlHs) e prontuários médicos, bem como a sua manutenção no Sistema. O diagnóstico de um caso de peste atualmente seria um evento inusitado e justificaria o envolvimento de diversos órgãos envolvidos no diagnóstico e controle de zoonoses, o que não ocorreu.

Conclusivamente, o diagnóstico da Peste deve estar 
devidamente fundamentado em evidências epidemiológicas, clínicas e microbiológicas ${ }^{1,3,11}$. A inexistência de casos corretamente diagnosticados, as notificações de falsos positivos e de registros indevidos de mortes e internações no SIS/SUS são um bom indicador do volume de deficiências acumuladas nas últimas décadas.

Cabe ao Estado, portanto, investir na capacitação dos profissionais que assistem nos diversos níveis assistenciais, bem como dos que respondem pelos laboratórios de análises clínicas, instando-os a observar as condutas constantes no Guia de Vigilância em Saúde e nos manuais específicos, editados pelo
MS, garantindo diagnósticos precoces e pronto tratamento ${ }^{3,6}$. Às Gerências de Vigilância em Saúde cumpre investigar todos os rumores acerca da ocorrência de doenças de notificação compulsória ${ }^{15}$ e, nesse caso, incentivar os Núcleos Hospitalares de Vigilância e as Comissões de Controle de Infecção Hospitalar a detectarem e investigarem o registro da CID A20 nas AIHs e prontuários médicos, se necessário, oferecendo assessoria técnica especializada, instando-os a notificá-los imediatamente à Secretaria Municipal de Saúde para o desencadeamento tempestivo de ações de controle, evitando-se que uma zoonose esquecida, que encarna o passado, cause sofrimentos e mortes à sociedade.

\section{REFERÊNCIAS}

1. Didier R, Mouffok N, Bitam I, Piarroux R, Drancourt M. Plague: history and contemporary analysis. J Infec 2013 Jan; 66(1): 18-26. doi:10.1016/j. jinf.2012.09.010

2. WHO. Plague around the world in 2019. World Health Organization. Weekly Epidemiological Record 94, 289-292. https://www.who.int/wer/2019/ wer9425/en/

3. Ministério da Saúde (BR). Secretaria de Vigilância em Saúde. Departamento de Vigilância Epidemiológica. Manual de vigilância e controle da peste. Brasília: Ministério da Saúde; 2008. 92 p. (Série A. Normas e Manuais Técnicos).

4. Coura JR, Silva JR, Oliveira Z, Lopes PFA. Focos inveterados de peste no Brasil. A propósito de um pequeno surto da doença ocorrido recentemente no município de Nova Friburgo, Estado do Rio de Janeiro. Rev Soc Bras Med Trop. 1967 Nov-Dez;1(6): 293-310. doi.org/10.1590/S0037-86821967000600002.

5. Sousa LLF, Alencar CHM, Almeida AMP, Cavalcanti LPG. Seroprevalence and spatial distribution dynamics of Yersinia pestis antibodies in dogs and cats from plague foci in the State of Ceará, Northeastern Brazil. Rev Soc Bras Med Trop, 2017 Nov-Dec; 50(6):769-776. doi: http://dx.doi.org/10.1590/0037-8682-02782017.

6. Tavares C, Aragão Al, Leal NC, Leal-Balbino TC, Oliveira MBM, Ferreira GMOG, Almeida AMP. The plague in Brazil: from now and then. In: Almeida AMP, Leal NC (eds.). Advances in Yersinia Research. New York: Springer; 2012. p. 69-77.

7. Stenseth NC, Atshabar BB, Begon M, Belmain SR, Bertherat E, Carniel E, et al. Plague: past, present and future. PLoS Med. 2008 Jan; 5(1): e3. https://doi. org/10.1371/journal.pmed.0050003.
8. Inglesby TV, Dennis DT, Henderson DA, Bartlett JG, Ascher MS, Eitzen E, et al. Plague as a Biological Weapon. Medical and Public Health Management. JAMA. 2000 May; 283(17): 2281-90. doi: 10.1001/jama.283.17.2281.

9. Cosgrove SE, Perl TM, Song X, Sisson SD. Ability of physicians to diagnose and manage illness due to category A bioterrorism agents. Arch Intern Med. 2005 Sep;165(17): 2002-6. doi:10.1001/archinte.165.17.2002.

10. Mead PS. Plague in Madagascar - A Tragic Opportunity for Improving Public. Health. N Engl J Med. 2018 Jan; 378(2): 106-8. doi: 10.1056/NEJMp1713881.

11. Butler T. Plague gives surprises in the first decade of the 21st century in the United States and worldwide. Am J Trop Med Hyg. 2013 Oct; 89(4): 788-93. doi:10.4269/ajtmh.13-0191.

12. Oliveira GM, Tavares C, Magalhães JLO, Almeida AMP. Peste, uma doença ocupacional. Rev Patol Trop. 2011 Jan-Mar; 40(1):15-22.

13. Tourdjman M, Ibraheem M, Brett M, DeBess E, Progulske B, Ettestad P, et al. Misidentification of Yersinia pestis by automated systems, resulting in delayed diagnoses of human plague infections - Oregon and New Mexico, 2010-2011. Clin Infect Dis. 2012 Oct; 55(7): e58-60. doi:10.1093/cid/cis578.

14. Chu M. Laboratory Manual of Plague Diagnosis Tests. Geneve: CDC/WHO; 2000. 129 p.

15. Brasil. Ministério da Saúde. Portaria de consolidação no 4 , de 28 de setembro de 2017. Consolidação das normas sobre os sistemas e os subsistemas do Sistema Único de Saúde. Diário Oficial [da] República Federativa do Brasil. 2017 Set 28; Seção 1. Lista Nacional de Notificação Compulsória (anexo 1 do anexo V, Portaria de Consolidação no 4 GM/MS de 3/10/2017). 